\title{
RETROSPECTIVE ANALYSIS OF 43 CASES OF INTRACRANIAL TUBERCULOSIS USING MR IMAGING
}

\author{
Liter Nguri'1, Pronami Borah², Rudra Kanta Gogoi ${ }^{3}$
}

${ }_{1}^{1}$ Postgraduate Trainee, Department of Radiodiagnosis, Assam Medical College, Dibrugarh.

${ }^{2}$ Associate professor, Department of Radiodiagnosis, Assam Medical College, Dibrugarh.

3Professor, Department of Radiodiagnosis, Assam Medical College, Dibrugarh.

\section{ABSTRACT}

\section{BACKGROUND}

Intracranial tuberculosis has varied presentation and if not treated early can lead to devastating clinical outcome. This study aimed at retrospectively analysing the varied forms of presentation of intracranial tuberculosis and its associated complications in our hospital setup.

\section{MATERIALS AND METHODS}

It is a descriptive type of study in which the MRI scans of 43 cases with intracranial tuberculosis, which were done in the Department of Radiodiagnosis, Assam Medical College, Dibrugarh, India, were retrospectively analysed. The study population comprised of 11 children and 32 adults with ages ranging from 11 to 60 yrs. (mean age, 26.58 yrs.). MR imaging was done by 1.5 Tesla machine using conventional spin echo sequences and additional sequences such as MR Spectroscopy and Diffusion-weighted Imaging.

\section{RESULTS}

It was found in this study that majority of cases of intracranial tuberculosis were in the age group of 11-20 yrs. (44.2\%). The most frequent tuberculous lesions encountered were tuberculomas $(79.1 \%)$ followed by tubercular meningitis $(72.1 \%)$, cerebritis (6.9\%) and abscesses (4.6\%). Tuberculomas were frequently located in cerebral hemispheres (88.2\%) followed by cerebellar hemispheres (61.7\%), brainstem (26.5\%) and basal ganglia (8.8\%). Tubercular Meningitis-induced infarcts were most commonly located in basal ganglia (73.3\%) followed by thalamus (33.3\%), brainstem (33.3\%), frontal lobe (33.3\%), internal capsule (26.7\%), cerebellar hemisphere (26.7\%), corpus callosum (20\%), parietal lobe (6.7\%) and external capsule (6.7\%). In adults, the most frequent lesions encountered in decreasing order of frequency were tuberculomas $(81.25 \%)$, tubercular meningitis (78.1\%), infarcts (31.25\%), cerebritis (9.37\%), abscess (6.3\%), ependymitis $(6.25 \%)$ and choroid plexitis (3.1\%). In children, the most frequent tuberculous lesions encountered were tuberculomas (72.7\%) followed by tubercular meningitis (54.5\%) and ependymitis $(9.0 \%)$.

\section{CONCLUSION}

MRI plays an important role in the diagnosis of intracranial tuberculosis and its associated complications. It helps in identifying the extent of involvement and differentiating tuberculous lesions from other pathologies.

\section{KEYWORDS}

Intracranial Tuberculosis, Tubercular Meningitis, Tuberculoma, MRI.

HOW TO CITE THIS ARTICLE: Nguri L, Borah P, Gogoi RK. Retrospective analysis of 43 cases of intracranial tuberculosis using MR imaging. J. Evolution Med. Dent. Sci. 2017;6(81):5682-5686, DOI: 10.14260/jemds/2017/1233

\section{BACKGROUND}

Tuberculosis is a common and deadliest disease in India accounting for 2.2 million cases out of 9.6 million global incidences according to WHO TB statistics of India for 2015.[1] CNS affection accounts for approximately $10 \%$ in all patients with tuberculosis.[2] CNS tuberculosis has varied manifestations including TB meningitis, tuberculomas, tubercular abscess, cerebritis, vasculitis and infarction. ${ }^{[3]}$

The aim of this study was to retrospectively analyse the varied presentations of intracranial tuberculosis using MRI scan in our hospital setup which will help making more confident diagnosis of intracranial tuberculosis in the future.

'Financial or Other Competing Interest': None.

Submission 17-08-2017, Peer Review 27-09-2017,

Acceptance 03-10-2017, Published 09-10-2017.

Corresponding Author:

Liter Nguri,

PNB (Paresh Nath Baruah) Ladies Hostel,

Assam Medical College, Dibrugarh.

E-mail: adiyanguri@gmail.com

DOI: $10.14260 /$ jemds/2017/1233

\section{MATERIALS AND METHODS}

Our department's records were reviewed from June 2016 to June 2017, and MRI scans of patients diagnosed with intracranial tuberculosis at our hospital were retrospectively studied. The institutional ethical committee approval was taken for the study. The study population comprised of 43 cases ( 11 children and 32 adults) with ages ranging from 11 to 60 yrs. (mean age 26.58 yrs.), suspected of suffering from intracranial tuberculosis based on clinical history and CSF analysis either before or after a diagnosis of intracranial tuberculosis was given on MRI scan. The known cases of treated intracranial tuberculosis/any brain space occupying lesions/previous history of stroke due to known vascular risk factors such as hypertension, diabetes and heart disease were excluded from the study.

The patients enrolled to the study were subjected to MRI using Siemens Magnetom AVANTO 1.5 TESLA machine. Head coil was used in all the patients in supine position. Conventional spin echo sequences, axial T1, T2 and FLAIR; Coronal T2; Sagittal T1; axial GRE (Gradient Recalled Echo); followed by post-contrast axial, coronal and sagittal images 
were obtained. Additional sequences like MR spectroscopy and DWI were acquired for tuberculous lesions whenever required. Multi-voxel spectroscopy data was acquired after contrast administration using a CSI sequence with TE 135 msec. Spectroscopy was avoided in small lesions which were in close proximity to the bone. Spectral heights of metabolites like choline, creatine, $\mathrm{N}$-acetyl aspartate, lactate, lipid and amino acids were studied. Metabolite ratios like $\mathrm{Cho} / \mathrm{Cr}$ and $\mathrm{NAA} / \mathrm{Cr}$ were also studied. As reference standards values of Cho/Cr $>1.5$ and NAA/Cr $<1.6$ were taken as abnormal which was similar to the study conducted by N Meena et al (2015).[4]

It is a descriptive type of study and patients' characteristics were presented using numbers and percentages. All the statistical calculations were done using SPSS (Statistical Package for the Social Sciences) 16.0.

All the cases were put on first line Antitubercular drugs and followed up clinically wherever possible. Isoniazid $(300$ $\mathrm{mg} / \mathrm{d})$, Rifampicin (10 mg/kg/d), Pyrazinamide $(30 \mathrm{mg} / \mathrm{kg} / \mathrm{d}$ in divided doses), ethambutol $(15-25 \mathrm{mg} / \mathrm{kg} / \mathrm{d}$ in divided doses) and pyridoxine (50 mg/d). In cases showing good response to Antitubercular drugs, ethambutol and pyrazinamide were discontinued and only isoniazid and Rifampicin were continued for 6 months. In cases with inadequate resolution of symptoms of meningitis, isoniazid and Rifampicin were prolonged for 9 - 12 months. In cases diagnosed with tubercular meningitis, Ceftriaxone (2 g IV twice daily), ampicillin (2 g IV four times daily and dexamethasone (8 $\mathrm{mg}$ IV twice daily) were administered. Dexamethasone was administered for 3 weeks and tapered over 3 weeks. In cases having infarcts, antiplatelet (aspirin 75 mg once daily) were administered. Majority of the patients showed improvement of symptoms after starting antitubercular treatment. 4 cases of TBM complicated by infarctions had poor prognosis despite adjunctive dexamethasone treatment.

\section{RESULTS}

Forty cases of intracranial tuberculosis were included in the study, out of which $32(74.4 \%)$ were adults ( $\geq 18$ yrs.) and 11 $(26.2 \%)$ cases were children ( $<18$ yrs.). Majority of cases were in the age group of $11-20$ yrs. (44.2\%). The most frequent tuberculous lesions encountered in our study were tuberculomas (79.1\%) followed by tubercular meningitis (72.1\%), cerebritis $(6.9 \%)$ and abscesses (4.6\%). The most frequent tubercular Meningitis-induced complications encountered were infarcts seen in 15 cases $(34.9 \%)$ and obstructive hydrocephalus in 15 cases (34.9\%). Tuberculomas were frequently located in cerebral hemispheres (88.2\%) followed by cerebellar hemispheres (61.7\%), brainstem (26.5\%) and basal ganglia (8.8\%) (Table/Fig. 1). Out of 34 cases which showed tuberculomas, caseating tuberculomas were seen in 27 cases $(79.4 \%)$ and non-caseating tuberculoma in 19 cases (55.8\%). Tubercular abscess diagnosis was made in $2(4.6 \%)$ cases and in both cases the lesion was located in the parietal lobe. Both cases of tubercular abscess showed elevated lipid/lactate peak and mildly elevated choline peak with no evidence of amino acid peak, thus excluding pyogenic abscess (Fig. 3). Tubercular Meningitis-induced infarcts were most commonly located in basal ganglia $(73.3 \%)$ followed by thalamus $(33.3 \%)$, brainstem (33.3\%), frontal lobe (33.3\%), internal capsule $(26.7 \%)$, cerebellar hemisphere $(26.7 \%)$, corpus callosum
(20\%), parietal lobe (6.7\%) and external capsule (6.7\%).

In adults, the most frequent lesions encountered in decreasing order of frequency were tuberculomas (81.25\%), tubercular meningitis (78.1\%) and abscess (6.3\%). The most frequent Meningitis-induced complications encountered in decreasing order of frequency were infarcts (31.25\%), obstructive hydrocephalus (25\%), cerebritis (9.37\%), ependymitis (6.25\%) and choroid plexitis (3.1\%) (Table/Fig. 2).

In children, the most frequent tuberculous lesions encountered were tuberculomas (72.7\%) followed by tubercular meningitis (54.5\%) and ependymitis (9.0\%). The most frequent tubercular Meningitis-induced complications in children were obstructive hydrocephalus (63.6\%) followed by infarcts (45.4\%) (Table/Fig. 3).

\begin{tabular}{|c|c|c|}
\hline Location & $\begin{array}{c}\text { Number } \\
\text { (n = 34) }\end{array}$ & Percentage \% \\
\hline Cerebral hemispheres & 30 & 88.2 \\
\hline Cerebellar hemispheres & 21 & 61.7 \\
\hline Brainstem & 9 & 26.5 \\
\hline Basal ganglia & 3 & 8.8 \\
\hline \multicolumn{2}{|c|}{ Table 1. Location of Tuberculomas } \\
\hline
\end{tabular}

\begin{tabular}{|c|c|c|}
\hline Lesion & $\begin{array}{l}\text { Number } \\
(n=32)\end{array}$ & Percentage \% \\
\hline Tuberculoma & 26 & 81.25 \\
\hline Tubercular Meningitis & 25 & 78.1 \\
\hline Tubercular Abscess & 2 & 6.3 \\
\hline Infarcts & 10 & 31.25 \\
\hline Obstructive Hydrocephalus & 8 & 25 \\
\hline Cerebritis & 3 & 9.37 \\
\hline Ependymitis & 2 & 6.25 \\
\hline Choroid Plexitis & 1 & 3.1 \\
\hline
\end{tabular}

\begin{tabular}{|c|c|c|}
\hline Lesion & $\begin{array}{l}\text { Number } \\
(n=11)\end{array}$ & Percentage $\%$ \\
\hline Tuberculoma & 8 & 72.7 \\
\hline Tubercular Meningitis & 6 & 54.5 \\
\hline Infarcts & 5 & 45.4 \\
\hline Obstructive Hydrocephalus & 7 & 63.6 \\
\hline Ependymitis & 1 & 9 \\
\hline \multicolumn{3}{|c|}{$\begin{array}{l}\text { Table 3. Types of Tuberculous Lesions } \\
\text { and Associated Complications in Children }\end{array}$} \\
\hline
\end{tabular}

\section{DISCUSSION}

Magnetic resonance imaging is highly sensitive in demonstrating intracranial tuberculous lesions accurately. Conventional MRI with additional techniques like MR spectroscopy and DWI provide an accurate assessment of various intracranial tuberculous lesions and associated complications. Overall tuberculous lesions of brain can be categorised into tuberculomas, tuberculous abscess and meningitis.

Sonmez G et al[5] retrospectively reviewed the data of 17 women and 10 men with age ranging from $14-51$ years (mean age of 26 years ) with intracranial tuberculomas. In our study, most of the cases were between the ages of $11-20$ yrs. (mean age of 26.6 yrs.) which closely coincides with the 
study conducted by Sonmez G et al.[5]

Kadriye Yasar et al[6] evaluated the radiological findings of 160 adult patients with tuberculous meningitis (TBM). Tuberculoma (37\%), basal meningitis (27\%), and hydrocephalus $(21 \%)$ were the most frequent signs found in the cranial CT or MRI scans. In our study, the most frequent tuberculous lesions encountered in adults in decreasing order of frequency were tuberculomas (81.25\%), tubercular meningitis (78.1\%) and abscess (6.3\%) which closely coincide with the findings given by Kadriye Yasar et al[6] (Fig. 4). In our study, hydrocephalus was included in one of the complications which resulted from tuberculous lesions such as basal meningitis or tuberculomas. The most common complications encountered in adults in decreasing order of frequency were infarcts $(31.25 \%)$, obstructive hydrocephalus (25\%), cerebritis $(9.37 \%)$ and ependymitis $(6.25 \%)$.

Farinha NJ et al[7] retrospectively studied 38 children with CNS tuberculosis. Out of 38 cases, 23 had tuberculous meningitis (TBM), 10 had tuberculous meningitis and associated tuberculomas and five had tuberculomas alone. In our study, the most common tuberculous lesions encountered in children were tuberculomas $(72.7 \%)$ followed by tubercular meningitis (54.5\%) and ependymitis (9.0\%) (Fig. 5) which were quite different from the results obtained in Farinha NJ et al study probably due to small number of cases in our study. In our study, the most common tuberculous meningitis-induced complications encountered in children were found to be obstructive hydrocephalus $(63.6 \%)$ followed by infarcts $(45.4 \%)$.

Sonmez G et al[5] retrospectively reviewed the data of 27 patients with intracranial tuberculomas and found out that out of total 64 tuberculomas found in these patients, 41 were distributed in the cerebral hemispheres, 17 in the cerebellar hemispheres, and 6 in the brainstem. In another retrospective study on 134 cases of clinically diagnosed TB patients conducted by $\mathrm{He} \mathrm{W}$ et $\mathrm{al},{ }^{[8]}$ brain parenchyma tuberculosis were detected more frequently in the cerebral hemispheres and cerebellar hemisphere. In our study, tuberculomas were found to be frequently located in cerebral hemispheres (88.2\%) followed by cerebellar hemispheres (61.7\%), brainstem (26.5\%) and basal ganglia (8.8\%) which was similar to the findings of Sonmez G et al.[5]

Tuberculomas have been grouped into three types based on MRI findings and include non-caseating, caseating with solid centre and caseating with central necrosis. ${ }^{[9,10]}$ Noncaseating tuberculomas usually show low signal intensity relative to brain parenchyma in $\mathrm{T} 1$ and $\mathrm{T} 2$ weighted images and homogenous enhancement on post-contrast scan. Caseating tuberculomas generally exhibit hypo-isointense signal intensity in T1 weighted images and iso-hypointense signal intensity in T2 weighted images on T2 weighted images with hyperintense centre in T2 weighted images in case of central necrosis (Fig. 2). These show ring like enhancement in contrast-enhanced images.[9-15] In studies conducted by Randy Jinkins J et al ${ }^{[14]}$ and McGuinness FE,[16] the MR signal characteristics of tuberculomas were described as follows:

\begin{tabular}{|c|c|}
\hline Tuberculoma & MRI \\
\hline Noncaseating & $\begin{array}{c}\text { Low signal intensity (SI) in T1 } \\
\text { High SI in T2 }\end{array}$ \\
\hline Caseating with solid centre & $\begin{array}{c}\text { Low to intermediate SI in T1 } \\
\text { Intermediate to low SI in T2 }\end{array}$ \\
\hline Caseating with liquid centre & Low SI in T1 \\
High SI in T2
\end{tabular}

In our study, out of 43 cases, tuberculomas were detected in 34 cases ( 26 cases in adults and 8 cases in children) and caseating tuberculomas were seen in 27 cases (79.4\%) and non-caseating tuberculoma in 19 cases (55.8\%). The tuberculomas found in our study were characterised as caseating and non-caseating based on MR signal characteristics which were similar to the findings of Misra UK et al[17] studied the pattern of stroke in tubercular meningitis and found that most of the tubercular related strokes were multiple, bilateral and located in the basal ganglia, anterior thalamus, anterior limb and genu of the internal capsule. The study also concluded that cortical stroke can also occur due to the involvement of proximal portion of the middle, anterior and posterior cerebral arteries as well as the supraclinoid portion of the internal carotid and basilar arteries. In our study Tubercular Meningitis-induced infarcts were most commonly located in basal ganglia $(73.3 \%)$ followed by thalamus (33.3\%) and brainstem (33.3\%) which were similar to the findings of Misra UK et al.[17] In our study, infarcts were also found in our frontal lobe (33.3\%), internal capsule (26.7\%), cerebellar hemisphere $(26.7 \%)$, corpus callosum (20\%), parietal lobe (6.7\%) and external capsule $(6.7 \%)$ which were different from the findings of Misra UK et al.[17]

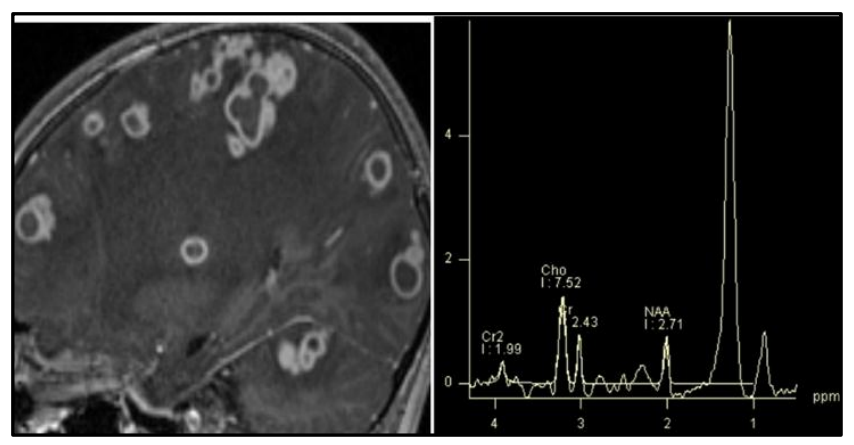

\section{Figure 1. Sagittal Post-contrast T1 Fat Saturated Image shows Multiple Peripherally Enhancing Discrete and Conglomerated Lesions}

MRS of the same case at TE 135 shows raised lipid peak, mildly elevated choline peak and decreased NAA peak.
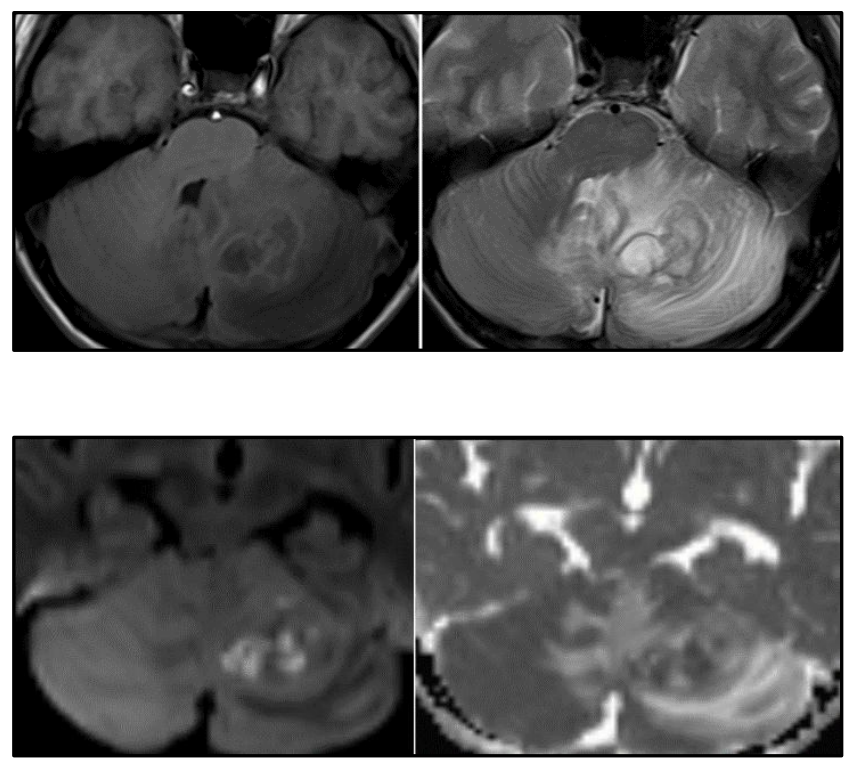


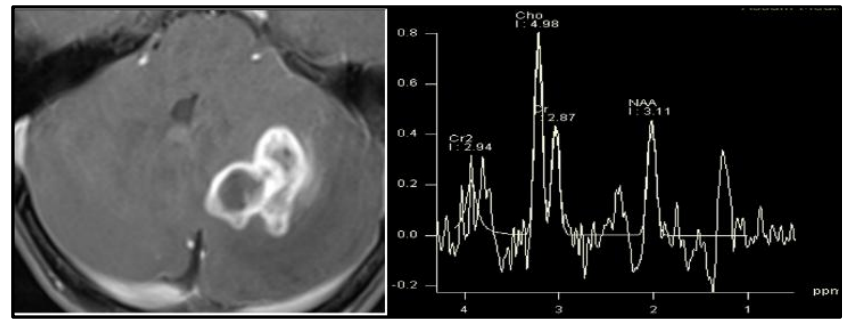

Figure 2. Necrotic Caseating Tuberculoma

Axial T1WI reveals few conglomerated hypointense lesions with peripheral hyperintense rim in left cerebellar hemisphere. Axial T2WI reveals hyperintense lesions with peripheral hypointense rim inciting moderate-to-severe perilesional oedema. DWI shows restricted diffusion with low ADC values. Axial post-contrast scan shows thick-walled peripherally enhancing conglomerated lesions, MRS shows elevated choline peak, lipid/lactate peak and mildly decreased NAA peak.
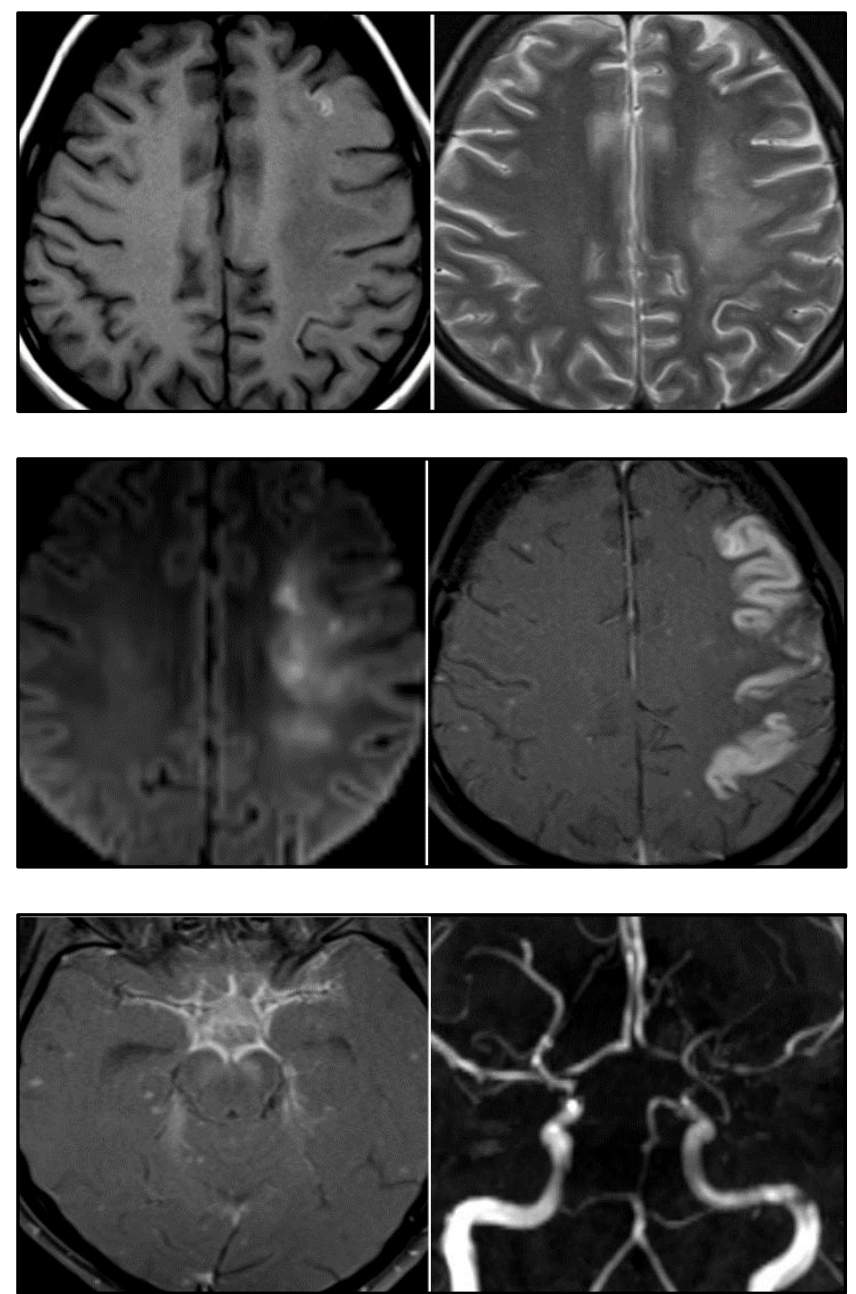

Figure 3. Meningitis-induced Vasculitis and Infarcts

Axial T1WI reveals hypointensity in subcortical white matter and corona radiata of left frontoparietal lobes, Axial T2WI shows hyperintensity in the affected regions, DWI shows restricted diffusion with low ADC values(not shown here),Axial post-contrast T1 FS images reveal gyriform cortical enhancement in the frontoparietal lobes and thickened meningeal enhancement in suprasellar cistern, interpeduncular cistern \& bilateral Sylvian fissure cisterns, TOF Angio reveals narrowing in proximal segment of left MCA.
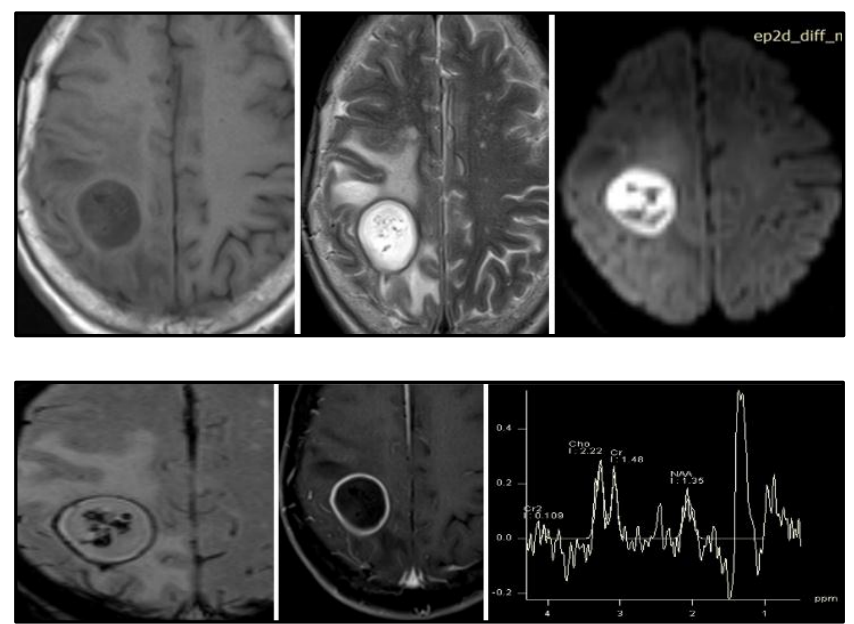

Figure 4. Tubercular Abscess

Axial T1 WI shows a well-defined hypointense lesion in right parietal lobe. Axial T2W1 shows hyperintense lesion with peripheral hypointense rim. DWI shows diffusion restriction in the lesion, GRE image shows peripheral and central blooming foci. Post-contrast scan shows thick-walled peripheral enhancement, MRS at TE 135 shows lipid/lactate peak, raised choline and decreased NAA peak.
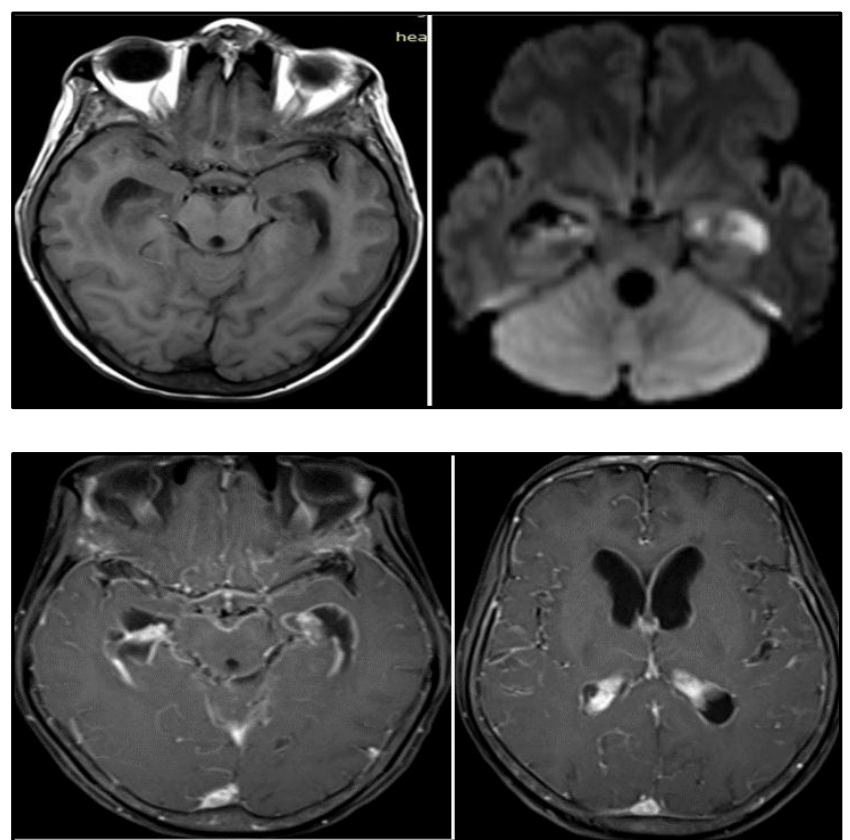

Figure 5. Meningitis with Ependymitis, Choroid Plexitis \& Communicating Hydrocephalus

Axial T1WI shows dilated bilateral lateral ventricles with dirty CSF picture in suprasellar cistern. Axial DWI shows restricted diffusion in bilateral choroid plexuses (>left) with dilatation of all the ventricles. Post-contrast scans reveal meningeal enhancement along the Sylvian fissures, interpeduncular, perimesencephalic cisterns, ependymal enhancement along bilateral ventricular linings and bilateral choroid plexus enhancement; TOF Angio reveals beaded appearance of bilateral MCA and PCA. 


\section{Limitation}

The findings of this study have limitations due to small number of patients with no confirmed histopathological diagnosis of tuberculomas and a lack of longterm followup. Despite these limitations, this study provides a useful insight into the most frequent lesions and associated complications of intracranial tuberculous.

\section{CONCLUSION}

MRI plays an important role in the diagnosis of intracranial tuberculosis and its associated complications. It helps in early diagnosis and management of meningitis thereby minimising potential life-threatening complications such as hydrocephalus and meningitis-induced infarcts. MRI also helps in identifying the extent of involvement and differentiating tuberculous lesions from other pathologies. Therefore, MRI should be the investigation of choice in a suspected case of intracranial tuberculosis whenever possible.

\section{REFERENCES}

[1] TB Statistics for India / National and state statistics. 2017. http://www.tbfacts.org/tb-statistics-india/

[2] Wood M, Anderson M. Neurological infections: major problems in Neurology. Chronic meningitis. Vol. 16. Philadelphia: WB Saunders 1998:169-248.

[3] Ahluwalia VV, Sagar DG, Singh TP, et al. MRI spectrum of CNS tuberculosis. JIACM 2013;14(1):83-90.

[4] Meena N, Bakshi VK, Kumar R, et al. Diagnostic role of magnetic resonance spectroscopy in intracranial space occupying lesions (IC-SOLs): a prospective analytical study. European Society of Radiology 2015;C-047.

[5] Sonmez G, Ozturk E, Sildiroglu HO, et al. MRI findings of intracranial tuberculomas. Clin Imaging 2008;32(2):88-92.

doi: 10.1016/j.clinimag.2007.08.024.

[6] Yaşar K, Pehlivanoglu F, Sengoz A, et al. Evaluation of radiological findings in 160 adult patients with tuberculous meningitis. Turk J Med Sci 2012;42(2):259-67.
[7] Farinha NJ, Razali KA, Holzel H, et al. Tuberculosis of the central nervous system in children: a 20 -year survey. J Infect 2000;41(1):61-8.

[8] He W, Ning F, Zhou X, et al. Magnetic Resonance Imaging features of parenchymal tuberculosis and dynamic evolution during antituberculous treatment. Analysis Chinese Journal of Tuberculosis and Respiratory Diseases (Zhonghua Jie $\mathrm{He} \mathrm{He} \mathrm{Hu}$ Xi Za Zhi) 2015;38(11):821-7.

DOI:10.3760/cma.j.issn.1001-0939.2015.11.006.

[9] Huang CR, Lui CC, Chang WN, et al. Neuro images of disseminated neurotuberculosis: report of one case. Clin Imaging 1999;23(4):218-22.

[10] Tsugawa J, Inoue H, Tsuboi Y, et al. Serial MRI findings of intracranial tuberculomas: a case report and review of the literature. No To Shinkei 2006;58(3):225-30.

[11] Kilani B, Ammari L, Tiouiri H, et al. Neuroradiologic manifestations of central nervous system tuberculosis in 122 adults. Rev Med Interne 2003;24(2):86-96.

[12] Kioumehr F, Dadsetan MR, Rooholamini SA, et al. Central nervous system tuberculosis: MRI. Neuroradiology 1994;36(2):93-6.

[13] Gupta RK, Jena A, Singh AK, et al. Role of magnetic resonance (MR) in the diagnosis and management of intracranial tuberculomas. Clin Radiol 1990;41(2):120-7.

[14] Jinkins JR, Gupta R, Chang KH, et al. MR imaging of central nervous system tuberculosis. Radiol Clin North Am 1995;33(4):771-86.

[15] Pui MH, Ahmad MN. Magnetization transfer imaging diagnosis of intracranial tuberculomas. Neuroradiology 2002;44(3):210-5.

[16] McGuinness FE. Intracranial tuberculosis. Clinical imaging in non-pulmonary tuberculosis. New York: Springer-Verlag Berlin Heidelberg 2000:5-25.

[17] Misra UK, Kalita J, Maurya PK. Stroke in tuberculous meningitis. J Neurol Sci 2011;303(1-2):22-30. 